\title{
EL SEMINARIO DE ORTEGA Y GASSET
}

El día 24 de febrero tuvo lugar la tercera sesión de nuestro Seminario sobre Ortega y Gasset. El texto elegldo para comentar era El tema de nuestro tiempa. Es este un texto sumamente interesante, en primer lugar porque es un texto que muchos intérpretes miran con clerto desagrado porque parece que se les resiste a una interpretación que pudiera ser encajada en el conjunto de la obra de Ortega. No hay que olvidar tampoco que el proplo Ortega dice en el "Prólogo para alemanes" que no estima este llbro. Pero en segundo lugar se trata de un texto que parece exponer quizás por primera vez con clerta precisión lo que Ortega va querer desarrollar en su vida filosofica. En él, por último, se plantea con contundencla la teoría de la verdad como resultado de la suma de perspectivas. Pero a mí desde hace muchos años me interesó este texto porque yo veía en él ya un claro adelanto a las tesis husserllanas de La crisis de las clencias europeas. Pues blen estos tres temas estuvieron presentes en las más de tres horas de discusión que duró el seminario, si blen el tema de la verdad y la perspectiva, con el tan traído y llevado tema del relativismo y valor de nuestras propuestas de verdad, se llevó una gran parte del tlempo.

El primer tema se refiere al lugar desde el cual interpretar el importante texto de Ortega, fundamentalmente porque ahi aparece una noción de vida que, como se vio a lo largo del Seminarlo, resulta sumamente problemática. ¿Es esa vida la menclonada en Meditaciones del Quijote? A la luz de los atrlbutos que parece aslgnarle Ortega no parece que sea así. Los rasgos blologiclstas, organiclstas que rezuma el texto no dejaron de ser motivo de escándalo para intérpretes anterlores, y tamblén ahora para algunos asistentes al Seminario esos rasgos invalldarían toda aproximación a este texto de Ortega desde la fenomenología. E.d. según una Interpretación convenclonal en este texto Ortega estaría deslumbrado por lecturas relativas a la blología, hasta el punto de que la noción de vida que aquí se maneja es fundamentalmente una vida de carácter orgánlco, por lo que difícllmente puede ser la vida como realidad radical que aparecerá, como veremos luego, en Qué es filosofía o la vida individual bajo cuya forma se nos da absoluta- 
mente todo, tal como se dice en Meditaciones del Quijote, y después, como se sabe, se repetirá en la recopilación de su pensamiento que hará Ortega en el "Prólogo para alemanes".

La polémica se centró fundamentalmente en si la vida es fundamentalmente vida subjetiva en el sentldo husserliano o es vida en el sentldo biológico, aunque sea una vida biológica de un carácter nuevo tal como Ortega se esfuerza por definir. La opinión mayoritaria fue que efectivamente los rasgos biológicos que configuran la vida en este texto pueden responder a una excesiva impostación de influencias de textos de biología, pero que en Ortega predomina el carácter histórico y el carácter subjetivo de la vida; el aspecto biológico estaría orientado a resaltar el carácter espontáneo que tiene la vida. Pero la vida humana es humana justo en la medida en que está también sometida al polo objetivo, siendo por tanto una vida sometida a la tensión o a la correlación sujeto-objeto. Es cierto que utillza Ortega un lenguaje sumamente biologlcista u orgánico, como cuando dice que pensar es pensar la verdad como digerir es asimilar los manjares. El pensamiento de la verdad sería una función de un órgano. Pero no parece que sea conveniente a una correcta hermenéutica esa Interpretación biológica. Más bien se trata de metáforas para captar la peculiaridad de la vida humana que es histórica y sometida a la correlación objetiva, por lo que la verdad no es algo coyuntural a la vida humana sino algo que le es esencial, e.d. sin lo cual la vida humana no es tal vida. En este sentido El Prof. Montero opinaba que la vida que aparece en este texto no es distinta de la vida radical de Qué es filosofía o de Meditaciones del Quijote.

Partiendo de esa opinión, que varios presentes se esforzaron por probar, se planteó la pregunta de si esa vida era la vida que aparece en el término Lebenswelt de Husserl. A esa pregunta el Prof. Montero contestó diciendo que era una pregunta difícil porque habia que decldir previamente con qué mundo de la vida husserllano queríamos comparar la vida y su mundo que aparece en este texto, puesto que en Husserl habia que distinguir tres nociones de Lebenswelt. De todas maneras ya de entrada quedó establecido algo cuya virtualidad saldría realmente en la tercera parte en la, desgraciadamente breve discusión de las ideas políticas con las que 
Ortega opera en este texto, a saber, que en El tema de nuestro tiempo se formulan tesis que adelantan Ideas básicas de la Krisis de Husserl, tales como el diagnóstico de la Edad Moderna, de la sensibilidad moderna, la suspicacia y el desdén hacia lo espontáneo e Inmediato, porque Descartes decidirá que "el verdadero mundo es el cualitativo, el geométrico". Pues bien, el objetivo del libro es mostrar el cambio de perspectiva necesario, la necesidad de volver a lo Inmediato, e.d. a la vida.

Justamente la exposición del Prof. Montero de la tripllcldad de la noción de mundo de la vida de Husserl dio ple para pasar al segundo gran tema, que se llevó más de una hora de discusión, en la que por otro lado se continuaba una polémica abierta en el Seminario anterior. En efecto, el Prof. Montero había comentado la existencia de tres nociones de Lebenswelt en Husserl, uno sería el que aparece en la $\mathrm{Kr} / \mathrm{s} / \mathrm{s}$, el mundo concreto histórico con sedimentos culturales; pero habría también un Lebenswelt, el de Experiencia y juicio, el de las experiencia de la subjetividad que construye ese mundo y el Lebenswelt primordial de la $V$ Meditación cartesiana, que es un mundo de la subjetividad radical que construye esas estructuras mundanas históricamente vinculantes. Tenemos pues un mundo cambiante según las culturas y unas estructuras previas o que condicionan lo histórico. Teniendo en cuenta el texto de Ortega, esto nos planteaba dos problemas básicos. Por un lado parecía rehacerse el camino de Husserl de resolver el psicologismo no mediante la postulación de un reino autónomo, sino retrotrayendo ese reino autónomo a la subjetividad, al fondo vital. Ahora bien, teniendo en cuenta la definición que Ortega hace de la verdad desde la noción de perspectiva, será necesario plantearse la pregunta por el carácter universalmente válido justamente de esas estructuras que Ortega va a tratar de definir como características de la vida humana. Mas si sólo históricamente tenemos acceso a ellas, ¿cuál es su carácter de verdad? ¿vale el carácter mismo perspectivístlco de la verdad que plantea Ortega comon aprlorl histórico o es exclusivamente un planteamiento del momento de Ortega? EI problema se plantea justamente en los términos en que Ortega enuncia la diferencla con otras épocas, en las que se creía que la verdad era válida para siempre, con la época actual, en la que se reconoce un carácter histórico a la verdad; pero entonces cabe la pregunta de si tiene cada época su 
verdad incomunlcable con las verdades de otras épocas o si para que sea verdad debe ser comunlcable con otras épocas, de modo que el Lebenswelt según la noción típica de la Krisis impllca que se deja traducir a otras culturas o no. Pues en la tesis de Ortega parecería estar implícito que sus propias teorias caen en el historicismo que enuncian, con lo que no valdrian como teorías fllosóficas.

La pregunta desató una vivísima polémica sobre el tema del apriori histórico él mismo ya no histórico y sobre nuestra posibllldad o pretensión de llegar a algo así; la polémica derivó incluso en un tema muy de actualidad, en la dificultad o incluso imposibilidad de practicar una comprensión - interpretación de pensadores o filósofos de otras época, Incluso en temas que parecerian aparentemente claros como pueden ser los principios lógicos. ¿Puedo suponer que otros pensadores utilizaron los mismos principios lógicos en el mismo sentido en que los utllizamos ahora?, se preguntaba el Prof. Montero con el decidido apoyo del Prof. C. Monedero. La seguridad de que el principio de no contradicción no significaba lo mismo para Kant, para Aristóteles y Parménides, parecía llevar a un relativismo; sin embargo el Prof. Montero terminaba asegurando que el pensamiento racional impllca aceptar que funcionamos como si esos principios fueran apriori, pero que de ahí no podemos pasar, con lo que se podría establecer una diferencia entre Husserl, en quien se podría decir que existe un fuerte apriorismo, mientras que en Ortega no lo habría.

El Prof. Vicent Martínez intentó mediar en la polémica recurriendo al 'principio de confianza' de Austin, que sería lo propio de un nivel, y la noción de ciencia reconstructiva de J. Habermas, que trataría en un segundo nivel de reconstruir esa justificación pragmática, reflexionándola y contándola desde el principio como un apriori para que haya comprensión, porque por muchas objeciones que podamos hacer a la traducción, en el fondo podemos traducir, lo que no sería posible si no admitiéramos algo en común. En mi opinión y así lo hice saber, la búsqueda de estas estructuras comunes que serían lo propio de la subjetividad en cuanto tal y por tanto apriori de comprensión y discurso es un objetivo decisivo de la fenomenología, que a mí me pareció ver expresado en la formulación husserliana de 
la Vorlesung de 1922, en la que se trata de convertir la reducción trascendental inicialmente ni eidética ni apodíctica, en reducción eidética y apodíctica, que no tiene tanto el sentido epistemológico con que Husserl la había podido concebir inicialmente cuanto el sentido de descubrir aquellas estructuras fundamentales de la subjetividad trascendental, sin las cuales no es pensable tal subjetividad. El Prof. Monedero se resistió a admitir la pretensión de un conocimiento de tal alcance cuando es cada persona la que "constituye" incluso el principio de contradicción, hasta el punto de que se daría una imposibilidad de entrar en la perspectiva del otro. Es cierto que el Prof. Conill advirtió reiteradamente contra el falibllismo de que todo puede resultar falsable, porque tal proposición caería sobre sus propias trampas; y si no todo es falsable, debemos sacar las consecuencias. No obstante, la polémica fue larga; Doña Pliar Allegue quiso remitirla a ya clásica y debatida polémica del innatismo o empirismo. Clertamente parece que nadie siguió esa vía, quizás porque tal vez esa polémica se debate en un terreno que en terminología husserliana se podría llamar de la "actitud natural', mientras que en nuestra discusión hablábamos más bien del sentido mismo que tiene el hablar, el poder traducir, el entendernos, en definitiva el compartir algo común, que yo creo que responde a esa idea husserliana de subjetividad trascendental.

Una pregunta punzante, como todas las suyas, fue la que el Prof. Zaccagnini, psicólogo de profesión, nos lanzó a los filósofos, y aunque aún la polémica anterior siguió ocupando una buena cantidad de tiempo, la pregunta mencionada sirvió para poder en su momento cambiar de tercio e Iniciar la última parte del comentarlo a El tema de nuestro tiempo. Es de suponer que comprenslblemente cansado de la larga discusión de los filósofos en si era o no posible pensar un aprlorl histórico, o si nuestras perspectivas de las cosas podían dar como resultado al go común, nos preguntó en realidad qué importancia tenía eso, qué consecuencias se derivaban de una postura u otra, porque parecía que en principio podía sencillamente dar Igual. Pues bien el Prof. García Baró introdujo la respuesta con gran precisión, pues el problema no es justamente un problema teórico, sino justo un problema práctico. En este sentido expliqué yo también el sentido práctico con el que Husserl inicia la Vorlesung a la que antes he aludido 
y al que también me he referido en el saludo de este mismo boletín. En cuestiones de racionalidad, de apoctlctlcldad no somos arbitrarios, no podemos declr lo que queramos, porque estamos sometidos, en opinión de Husserl, a normas que constituyen justamente nuestra autonomía. Frente a ellas ningún poder fáctico, auch kein Gott, como dice Husserl en esa Vorlesung podría nada. La racionalidad, terminó diciendo el Prof. Montero, no la podemos evitar, pero tampoco puedo afirmar que lo que yo encuentro como necesario y universal p.e. para ser libre, lo sea así de un modo absoluto para todos. A mi parece que la cuestión tiene su importancia en periodos de crisis, que es Justo cuando pensaron tanto Husserl como Ortega, porque en base a esas discusiones teóricas, Husserl pudo anticipar un repudio del nacionalismo.

¿Y Ortega? ¿cuál es la postura política de Ortega en este texto? La pregunta no carece de alcance, porque en este texto Ortega se va a adelantar claríslmamente al Husserl de La crisis de las ciencias europeas, en primer lugar al diagnosticar la crisis que estaba padeciendo Europa y que era una crisis consecuente a la ideas procedentes de la Edad Moderna; y en segundo lugar, al pedir que Justamente el tema de nuestro tiempo era el volver la razón, la ideas, a la vida, e.d. reconducirlas a lo Inmediato, diríamos en terminología husserliana, reconducirlas al mundo de la vida. Expresamente pregunté qué podía suponer esto de terapia política, de idea política. Tanto el Prof. Montero como el Prof. Conill formularon el pensamiento de Ortega en ese sentido como la petición de revitalizar todas las esclerotlzaclones ideológicas o p.e. las prácticas políticas, tales como la democracia que fácilmente pueden separarse de la vida y convertirse en dogmas en los que se cree como en la Virgen del Pilar; mas entonces se convierten en instancias ajenas a los individuos, perdiendo su vitalidad.

No dejó de faltar polémica tampoco en este punto, pues una vez más no queda claro cuál es el ideal ortegulano en la propuesta de revitalizaclón, Justamente porque la idea de vida y por tanto los valores vitales que hay que insuflar para revitalizar las esclerotizaciones no parecen excesivamente adecuados, como observó D. Agustín Serrano de Haro. Sin embargo, a mí me parece que las ideas políticas de Ortega en este texto deben ser también 
leidas dentro del contexto de su obra, interpretando la vida desde la noción de realidad radical, tal como lo hemos explicado un poco más arriba. De todas maneras creo que la sesión del Seminario sirvio para subrayar la Importancia de este texto tanto por lo problemas que trata como por lo que significa en la fenomenología de anticipación de fórmulas que Husserl expondrá en el libro que en la actualidad más se estima del fundador de la fenomenología. Esta coincidencia no creo que deba ser cltada para estimular ningún orgullo necio, sino para mostrar la necesidad de enmarcar a Ortega dentro de la fenomenología, lo que de nuevo tuvimos ocasión de ver en la siguiente sesión de Seminario.

En efecto, la cuarta sesión del Seminario que tuvo lugar el día 5 de mayo estuvo dedicada al importantísimo libro de Ortega Qué es filosofía. Un aura especial rodea a este libro. Es sabido que el libro procede de un curso que Ortega empezo a leer en la Facultad de Fiiosofía pero que no pudo continuar por problemas políticos, por lo que tuvo que trasladarse a un cine. El éxito del curso fue tal que pronto tuvieron que cambiar de local porque el inicialmente previsto no podía albergar a todos los asistentes. Pero independientemente de esta circunstancia el libro tlene otros méritos suficientemente llamativos como para merecer una consideración explícita. En ese sentido creo que nuestro Seminarlo no defraudo. Empecemos diciendo que la asistencia al mismo fue considerable. También nuestro Seminario consigulo llenar el aula en el que lo comenzamos, sin que por otro lado esa clrcunstancia variara la animación de la discusión, en la que se pusieron de manifiesto aspectos de este libro que pueden servir de excelentes motivos de investigaciones futuras. Desde esta conslderación creo que en general nuestro Seminario va a descubrir cantidad de motivos, que por no haber sido Ortega cuestionado, en el sentido etimológico, en nuestro país desde la perspectiva del movimiento fenomenologico al que pertenece, no habian sido antes puestos sobre el tapete de la discusión.

El Seminarlo tuvo tres partes, en las que se tocaron diversos temas dependientes de la pregunta con la que el Prof. Montero abrió la sesión de por qué Ortega deja de hablar de subjetividad para hablar de vida, con lo que se ponía sobre el tapete la relación de Ortega con Husserl; y en segun- 
do lugar el problema de la interpretación del método fenomenológico de Ortega desde la estructura misma de la obra Qué es filosofía, lo que suscitó discusiones sobre el lugar del método en la filosofía orteguiana. Así la primera hora se dedicó a centrar los temas de discusión, que se dirigió a estudiar el papel del concepto de la actitud natural, porque parecía que la noción orteguiana de vida podría estar más cercana al concepto ordinario que tenemos de nuestra vida, que al husserliano de subjetividad pura.

La segunda hora transcurrió en una discusión entre quienes defendían la vinculación de Ortega al proyecto husserliano y quienes lo desvinculaban o resaltaban las fuertes diferencias entre ambos modelos. Hasta cierto punto también dependía la defensa de lo uno y lo otro del concepto que se tuviera de actitud natural y de su papel en la fenomenología; por lo que se toco el tema de los diferentes sentidos de la actitud natural en Husserl y consecuentemente en Ortega. También fue necesario, para centrar la discusión, volver con cierto detenimiento a la presentación de la estructura del libro comentado, donde se veía la similitud y el adelanto que ortega hace de temas que Husserl desarrollará posteriormente en la crisis de las ciencias europeas.

En fin, la tercera parte, $y$ al hilo de la pregunta sobre el lugar del futuro como determinante en la estructura del tiempo, llevó a relativizar la influencia de Heidegger incluso en esa adscripción; pero justamente en ese contexto volvió a aparecer la importancia que ortega daría de entrada a un sistema de preferencias como apriori en la vida, a diferencia de Husserl que antes que la práctica pondría la teoría, lo que por supuesto fue motivo también de discusión.

Tres fueron por tanto los grandes temas que se tocaron. En primer lugar fue importante el tener en cuenta la estructura misma de la obra comentada, siguiendo el mismo símil orteguiano de los círculos o navegaciones. Nos encontramos entonces con que el viaje no es muy diferente del recorrido por Husserl en la Krisis pero realizado por nuestro autor unos cuantos años antes, lo que da a Ortega un lugar preferente en la fenomenología. En efecto, se mostró que Qué es filosofía empieza con un análisis del 
significado de la física y de la cultura determinada por la física, lo que llevaba al exterminio o mínima expresión de la filosofía, por lo que era necesario constatar la dependencia que ese mundo de la física tiene del mundo ordinario del científico, de una vida anterior solo en la cual tiene sentido. Después vendrá la discusión de lo que Husserl llamará el principio de los principios, para en la tercera parte exponer la necesidad de poner ante todo como hecho o realidad radical la vida sólo en la cual el mundo tiene sentido. La última parte del libro, ya dedicada al análisis de lo que Ortega llamará la tierra Ignota se dedicará al estudio y exposición de las categorías de esta vida, donde parece hacerse presente la influencia de Heidegger.

Pero esa estructura plantea dos problemas, uno el metodológico, en la pregunta propuesta por el Prof. Conlll de si el método que en la estructura parece asumir cierto papel dirigente, agota el planteamiento ortegulano o si más bien no es sino un punto de partida que deberá abrirse a un planteamiento más hermenéutico, con lo que tendríamos una de las razones de por qué vida y no subjetividad. La superación de la modernidad sería entonces en Ortega un tema fundamental. La propia crítica al sustanciallsmo cartesiano asume la importancia de representar la superación de todo un modelo de filosofía, en la que por cierto estaría la fenomenología muchas veces prendida. El otro problema que plantea esa estructura es el del carácter de la vida a la que llegamos y su relación con la actitud natural. En efecto, al Prof. VIcent Martínez le parecía excesivamente optimista la adscripción de Ortega a la fenomenología, justamente porque Husserl exigiría la superación de la actitud natural, mientras que Ortega trata de volver a la vida tal como esta vive ejecutivamente en la actitud natural. Un recorrido por los textos críticos de Ortega respecto a la fenomenología nos daría la clave de por qué vida y no subjetividad trascendental.

Sin embargo, el concepto de actitud natural no es Husserl un concepto sin densidad, pues, como yo mismo expuse, es posible distinguir en Husserl hasta cuatro conceptos de actitud natural. En Ortega, justamente en este texto, podemos hallar tres. El tercero, la actitud natural del yo recuperada tras la reducción, e.d. la vida ordinaria del yo, pero después de practicar 
la reducción, que según el Prof. García Baro, habría que ver hasta qué punto es 'actitud natural', ¿coincide con la vida descubierta por Ortega? A D. Agustín Serrano de Haro le parece que el parecido es mínimo, incluso habría una importante diferencia en la concepción del hecho fundamental, pues mientras para Husserl la relación que une los elementos del apriorl universal de correlación, la subjetividad y el mundo, es una relación bilateral, para Ortega esa relación sería unilateral, aunque 'mutua', porque es cierto que sin objeto no hay sujeto y sin sujeto no hay objeto, porque el objeto agota su ser en el aparecer. Para Husserl la dependencia es de correlación, lo que sería absolutamente desconocido en la actitud natural, por eso la cercanía de ambos pensadores sería sólo aparente.

El Prof. Rodríguez Rlal defendio, en contestación tanto a la postura del Prof. V. Martínez como a la de D. A. Serrano de Haro, la equivalencia del proyecto fenomenológico de Ortega con el de Husserl. Incluso defendería, contestando también a una incitante cuestión del Prof. Zaccagnini, que la investigación trascendental, e.d. aquella que va a la realidad más profunda, que tiene que traspasar la costra primera de la vida para liegar al fondo de la realidad última, que es como Ortega plantea su investigación, es una investigación eidética, que busca las estructuras generales de la vida humana, estructuras que, por otro lado, como nos citaba el mismo profesor, están según Ortega a la base de cualquier antropología. No dejó de recordar en este contexto el Prof. Rodríguez Rlal la carta de Husserl a Roman Ingarden comentando la visita que le hizo Ortega en 1934 en la que el filósofo español aparecía como director de una Escuela de fenomenología en Madrid. Llama la atención que Ortega nunca se presentara en España como fenomenólogo, lo que sin lugar a dudas provocó graves distorsiones en la interpretación de su pensamiento, de las cuales todavía estamos viviendo.

Sin embargo, como había dicho el Prof. ZaccagnIni, Ortega no es fenomenólogo porque nosotros lo digamos ni porque nosotros hagamos de él una lectura fenomenológica. Deberemos mostrar la semejanza de estructura de sus filosofías o de sus conceptos y siguiendo el planteamiento del prof. Montero, el Prof. García Baró planteó la diferencia en términos muy precisos, partiendo, es cierto, de los análisis de Husserl en las Ideas, e incluso en 
la $\vee$ Meditación cartesiana, donde la reducción primordial, e.d. aquella reducción que, realizada dentro de la reducción trascendental, nos ha de dar el mundo primordial. Pues bien, este mundo primordial es el mundo de la naturaleza no diferente del mundo previsto en las Ideas, e.d. un mundo de actos ponentes pertenecientes al ámbito de lo teórico, donde el interés, la teleología, por tanto los valores y la estimativa, como dirá el Prof. Montero, no pertenecen en la fenomenología Inicial de Husserl, al núcleo noemático sino como capas periféricas posteriores. A diferencia de este Husserl, en el que lo práctico, sería secundario, la vida de Ortega, quizás bajo la influencia de Scheler, es un haz de preferencias, que constituyen un aprlori para el conocimiento, que por tanto está sentimentalmente modulado. En este sentido la vida orteguiana estaría lejos de la subjetividad trascendental husserliana, que según Montero Mollner, se habría centrado en el periodo de su configuración como fenomenología trascendental hasta Ideas, como una subjetividad atenta a las estructuras lógicas, descuidando la vertiente axiológica y estimativa, por tanto práctica de la vida.

Clerto es que quien esto escribe no siempre estuvo de acuerdo con esta postura de los Profesores Montero y García Baro, porque, según Husserl, el mundo de la vida es un mundo de slgnificatlvidad práctica, en el que la práctica precede a la teoría. Sólo que el análisis de Ideas es un análisis que se centra en los actos de la conciencia, cuando esos actos de la conciencia viven de un mundo de significatividad plena en el que la percepción aislada no tiene sentido. Para el Prof. Montero en Husserl el carácter de signiflcativldad del mundo está claramente presente en el último Husserl, desde que en las Meditaciones cartesianas aparece el mundo concreto de la vida; lo que Husserl no haría sería revisar desde la nueva luz la anterior comprensión de la subjetividad desde las categorías más centradas en la lógica pura, para adecuarlas al nuevo nivel; de hecho nos recordaba el Prof. Montero que el primer disgusto que scheler le causó a Husserl fue el decirle que el bonun precede el verum. También el Prof. García Baró recordó que Husserl al nivel de las LECCIONES sobre filosofía primera recuperará el nivel teleológico, así como también Flnk recordará sobre todo en los bocetos para la revisión de la I Meditación cartesiana el interés que actúa en el conocimiento. La conclusión de todo esto es de todos modos que 
la noción de vida orteguiana definida además como quehacer está orientada a la acción, es una vida práctica.

Justamente esta cuestión tratada en Qué es filosofía en las últimas lecciones sobre los atributos de la vida ocupó la última hora. En ella e efecto aparece en primer término la posible Influencia de Heidegger. Pues si hasta esa lección la presentación de qué es la filosofía se ha hecho según el modelo husserllano, incluso como hemos dicho de una forma que se adelanta a la husserliana de la $\mathrm{Kr} / \mathrm{sis}$, en la exposición de los atributos de la vida parece en principio depender de Heidegger. Así nos dice tajantemente que la vida es futurición, dando entonces un predominio, un carácter dirigente en la configuración de la vida al futuro, de modo semejante a como lo hace Heidegger. El tema tiene interés pues es Importante justo para evaluar la influencia de Heidegger. A este respecto opinaba primero el Prof. Rodríguez Rlal que al haberse decidido Ortega por la historicidad mucho antes de que pudiera leer Ser y tiempo, la temporalidad de la vida ya era para Ortega un atributo de la misma. El problema sin embargo, para mí, no era ese, sino qué instante del tiempo tenía el carácter rector en la temporalidad. El Prof. Montero creía recordar que Ortega había asumido ya por entonces la tesis de Bergson de que frente a una temporalidad mostrenca y muerta, la de lo hecho, él se decantaba por una temporalidad de la creatividad, del futuro, con lo que no dependería de Heidegger.

Muy vivo fue el debate de estos extremos; en conjunto parecía que el hecho de que la vida fuera biográfica daba al futuro un papel importante; sin embargo, tampoco había que exagerar ese papel, pues en la dinámica entre vocación y destino, el futuro es un campo de posibilidades no arbitrario porque está determinado por lo que somos, por el pasado. El pasado es nuestra circunstancia, a partir de la cual decidimos nuestro futuro. Por eso habría que matizar las frases orteguianas de este texto en el contexto más amplio de su desarrollo, donde el yo es preocupación de futuro, pero no la vida, porque el yo es sólo un momento de la vida. 